\title{
Article
}

\section{The Association between Nutritional Status and In-Hospital Mortality of COVID-19 in Critically-Ill Patients in the ICU}

\author{
Michał Czapla ${ }^{1,2}$ (D), Raúl Juárez-Vela ${ }^{3, *(D)}$, Vicente Gea-Caballero ${ }^{4}$ D , Stanisław Zieliński ${ }^{5,6}$ \\ and Marzena Zielińska ${ }^{5,7}$
}

check for updates

Citation: Czapla, M.; Juárez-Vela, R.; Gea-Caballero, V.; Zieliński, S.;

Zielińska, M. The Association between Nutritional Status and InHospital Mortality of COVID-19 in Critically-Ill Patients in the ICU. Nutrients 2021, 13, 3302. https:// doi.org/10.3390/nu13103302

Academic Editors: Dimitrios T. Karayiannis and Zafeiria Mastora

Received: 30 August 2021

Accepted: 19 September 2021

Published: 22 September 2021

Publisher's Note: MDPI stays neutral with regard to jurisdictional claims in published maps and institutional affiliations.

Copyright: (c) 2021 by the authors. Licensee MDPI, Basel, Switzerland. This article is an open access article distributed under the terms and conditions of the Creative Commons Attribution (CC BY) license (https:// creativecommons.org/licenses/by/ $4.0 /)$.
1 Department of Public Health, Faculty of Health Sciences, Wroclaw Medical University, 51-618 Wroclaw, Poland; michal.czapla@umed.wroc.pl

2 Institute of Heart Diseases, University Hospital, 50-566 Wroclaw, Poland

3 Biomedical Research Centre of La Rioja (CIBIR), Research Group GRUPAC, Research Unit on Health System Sustainability (GISSOS), University of La Rioja, 26004 Logroño, Spain

4 Faculty of Health Sciences, International University of Valencia, 46002 Valencia, Spain; vicenteantonio.gea@campusviu.es

5 Department and Clinic of Anaesthesiology and Intensive Therapy, Faculty of Medicine, Wroclaw Medical University, 50-556 Wrocław, Poland; stanislaw.zielinski@umed.wroc.pl (S.Z.); marzena.zielinska@umed.wroc.pl (M.Z.)

6 Department of Anaesthesiology and Intensive Care, University Hospital, 50-556 Wrocław, Poland

7 Department of Paediatric Anaesthesiology and Intensive Care, University Hospital, 50-556 Wrocław, Poland

* Correspondence: raul.juarez@unirioja.es

Abstract: Background: Coronavirus disease 2019 (COVID-19) has become one of the leading causes of death worldwide. The impact of poor nutritional status on increased mortality and prolonged ICU (intensive care unit) stay in critically ill patients is well-documented. This study aims to assess how nutritional status and BMI (body mass index) affected in-hospital mortality in critically ill COVID-19 patients Methods: We conducted a retrospective study and analysed medical records of 286 COVID-19 patients admitted to the intensive care unit of the University Clinical Hospital in Wroclaw (Poland). Results: A total of 286 patients were analysed. In the sample group, $8 \%$ of patients who died had a BMI within the normal range, $46 \%$ were overweight, and $46 \%$ were obese. There was a statistically significantly higher death rate in men (73\%) and those with BMIs between 25.0-29.9 ( $p=0.011)$. Nonsurvivors had a statistically significantly higher HF (Heart Failure) rate $(p=0.037)$ and HT (hypertension) rate $(p<0.001)$. Furthermore, nonsurvivors were statistically significantly older $(p<0.001)$. The risk of death was higher in overweight patients $(\mathrm{HR}=2.13 ; p=0.038)$. Mortality was influenced by higher scores in parameters such as age $(\mathrm{HR}=1.03 ; p=0.001)$, NRS2002 (nutritional risk score, $\mathrm{HR}=1.18 ; p=0.019)$, PCT (procalcitonin, $\mathrm{HR}=1.10 ; p<0.001)$ and potassium level $(\mathrm{HR}=1.40$; $p=0.023$ ). Conclusions: Being overweight in critically ill COVID-19 patients requiring invasive mechanical ventilation increases their risk of death significantly. Additional factors indicating a higher risk of death include the patient's age, high PCT, potassium levels, and NRS $\geq 3$ measured at the time of admission to the ICU.

Keywords: COVID-19; malnutrition; SARS-CoV-2; nutritional status; intensive care unit

\section{Introduction}

On 30 August 2021, 216 million people were infected, and 4.5 million had died of severe coronavirus disease 2019 (COVID-19) [1]. The impact of poor nutritional status on increased mortality and prolonged ICU (intensive care unit) stays in critically ill patients is well known and well-documented [2]. Old age, male sex, comorbidities, being overweight, obesity and malnutrition are some of the known risk factors for severe COVID-19 cases [3]. Moreover, COVID-19 infection lasting several days or even weeks prior to ICU admission enhances patient malnutrition, which in turn leads to increased pathogenicity of the infecting agent and disease progression [4,5]. Furthermore, published studies show a risk of 
malnutrition in COVID-19 patients. The incidence of malnutrition in patients hospitalised for COVID-19 is 50\% [6,7]. Due to malnutrition observed in mechanically ventilated patients even months after discharge from the ICU, both the examination of the nutritional status of critically ill patients at the beginning of hospitalisation and early initiation of nutritional treatment are of great importance [8]. Being overweight and obesity are also factors that worsen prognosis. Individuals coping with these conditions have a higher risk of CVD (cardiovascular disease) and DM (diabetes mellitus). In addition, overweight and obese individuals may experience respiratory complications due to increased ventilatory demand, increased work during breathing, respiratory muscle insufficiency and decreased respiratory compliance [9].

Patients who require treatment in the ICU should be assessed for nutritional status [10]. According to the criteria for diagnosing malnutrition set out by the Global Leadership Initiative on Malnutrition (GLIM), every patient at increased risk for malnutrition should be screened. GLIM indicates that tools such as NRS 2002 (Nutritional Risk Score), SGA (Subjective Global Assessment), MUST (Malnutrition Universal Screening Tool), or MNA (Mini Nutritional Assessment) can be used for screening. In Poland, every person admitted to a hospital ward should undergo a screening test performed using NRS 2002 or SGA (the test does not apply to patients in a hospital Emergency Department; ED) [11,12]. According to ESPEN (European Society for Clinical Nutrition and Metabolism) experts, at-risk patients with more severe COVID-19 should be screened with NRS 2002 if hospitalised [13].

This study aims to assess how nutritional status and BMI (body mass index) affected in-hospital mortality in critically ill COVID-19 patients.

\section{Materials and Methods}

\subsection{Study Design and Setting}

A retrospective study was performed based on an analysis of medical records of active COVID-19 patients (ICD10: U07) admitted to the ICU of the University Clinical Hospital in Wroclaw (Poland) between September 2020 and June 2021. The study followed the STROBE (Strengthening the Reporting of Observational Studies in Epidemiology) guidelines.

\subsection{Study Population}

All the patients who met the following inclusion criteria were analysed: primary diagnosis of COVID-19 (confirmed by RT-PCR), age $\geq 18$ years, mechanical ventilation (invasive ventilation), hospitalisation in the ICU.

A final group of 286 patients' medical records was analysed. The analysis included data (collected at the time admission) concerning patients' age, sex and BMI; test results such as total cholesterol (TC), triglycerides (TGs), albumins, lymphocytes, potassium, sodium, C-reactive protein (CRP), procalcitonin (PCT); data concerning medical history and comorbidities, as well as assessment of the patients' nutritional status using NRS 2002.

\subsection{Nutritional Screening}

The NRS 2002 is one of the screening tools recommended by GLIM for risk assessment of nutritional status. The scale consists of two parts.

1. Impaired nutritional status, in which weight loss and BMI are assessed. The same applies to the percentage of food intake compared to its requirements within the last week. The rating scale is $0-3$ points, where 0 is lack of deterioration of health status, and 3 is severe deterioration of health status.

2. Severity of disease (an increase in requirements), in which, depending on the disease, patients may receive $0-3$ points, where 0 is normal nutritional requirements and 3 is high disease severity (e.g., head injury, bone marrow transplant). Moreover, if patients are over 70, they get an additional point. Thus, patients can score $0-7$ points. Nutritional therapy is indicated in patients with NRS $\geq 3$ [14]. The WHO criteria were used for classifying patients as underweight (BMI $<18.5 \mathrm{~kg} / \mathrm{m}^{2}$ ), with normal weight (BMI: $18.5-24.9 \mathrm{~kg} / \mathrm{m}^{2}$ ), pre-obese (BMI: $25-29.9 \mathrm{~kg} / \mathrm{m}^{2}$ ) and obese (BMI $\geq 30 \mathrm{~kg} / \mathrm{m}^{2}$ ). 
A physician measured the NRS 2002 and BMI at the time of admission to the ICU.

\subsection{Statistical Analysis}

Statistical analysis was performed using Statistica 13.1 software (TIBCO, Inc., Palo Alto, CA, USA). First, arithmetic means and standard deviations were calculated for measurable variables. Next, quantitative variables were examined using the Shapiro-Wilk test to determine the distribution type. Then, intergroup comparisons were made using the $t$-test or the Mann-Whitney $U$ test (if assumptions were met). Finally, the comparison of results of more than two groups was performed using one-way analysis of variance (ANOVA) or the Kruskal-Wallis test (if assumptions were met).

Survival analysis was carried out using the Kaplan-Meier method and refers to ICU mortality. The log-rank test was used for comparing patient survival against selected clinical variables. The Cox proportional hazards model was used for assessing the influence of qualitative or quantitative variables on patient survival. The analysis included both categorical variables and continuous variables. The categorical variables included sex, BMI (18.5-24.9, <18.5, 25.0-29.9, $\geq 30$ ), NRS (<3 vs. $\geq 3$ ), HF (heart failure, yes/no), HT (hypertension, yes/no), DM (diabetes mellitus, yes/no), CVD (cardiovascular disease, yes/no), CKD (chronic kidney disease, yes/no), CRD (chronic respiratory disease, yes/no), TGs (triglycerides, <135, 135-200, >200), TC (total cholesterol, $<40,>40$ ). The continuous variables included age, BMI $\left[\mathrm{kg} / \mathrm{m}^{2}\right]$, height $[\mathrm{m}]$, body weight $[\mathrm{kg}]$, TGs $[\mathrm{mg} / \mathrm{dL}]$, TC $[\mathrm{mg} / \mathrm{dL}]$, CRP $[\mathrm{mg} / \mathrm{L}]$, albumins $[\mathrm{g} / \mathrm{dL}]$, lymphocytes [\%], PCT [ng/mL], potassium $[\mathrm{mmol} / \mathrm{l}]$, sodium $[\mathrm{mmol} / \mathrm{L}]$.

Variables such as BMI, TC, or NRS were analysed as continuous and categorical variables in the univariate model. Variables were included in the multivariate model in accordance with the adopted criteria. Those criteria included the outcome of $p<0.30$ in a univariate model, a lack of correlation of variables, and clinical recommendations. Multivariate analysis was performed using backwards elimination to stay in the model. For the final multivariate model, the variables were selected according to the better fit of the model based on the assessment of the goodness of fit (AIC). The results were considered statistically significant at $p<0.05$.

\section{Results}

\subsection{Characteristics of the Group}

The profile of the whole group with a comparison of the analysed characteristics of the survivors and nonsurvivors is shown in Table 1. A total of 286 patients were analysed. Due to a lack of data for some parameters, those numbers are smaller and are provided for each variable. There was a statistically significantly higher death rate in men $(73 \%, n=142)$ and those with BMI between $25.0-29.9(46 \%$ vs. $26 \% ; p=0.011)$. Nonsurvivors had a statistically significantly higher HF rate ( $9 \%$ vs. $2 \% ; p=0.037)$ and HT rate $(55 \%$ vs. $24 \% ; p<0.001)$. Furthermore, nonsurvivors were statistically significantly older $(\overline{\mathrm{x}}=63.6$ vs. $\overline{\mathrm{x}}=53.8$ years; $p<0,001)$, taller $(\overline{\mathrm{x}}=175.9$ vs. $\overline{\mathrm{x}}=172.1 \mathrm{~cm} ; p=0.008)$. Considering laboratory test parameters, PCT levels were statistically significantly higher in nonsurvivors. TC levels were statistically significantly lower in nonsurvivors (Table 1). 
Table 1. Characteristics of the group with a comparison of survivors and nonsurvivors.

\begin{tabular}{|c|c|c|c|c|c|c|c|c|}
\hline \multirow{2}{*}{\multicolumn{2}{|c|}{ Variables }} & \multirow{2}{*}{\multicolumn{2}{|c|}{ Total $(n=286)$}} & \multicolumn{4}{|c|}{ Death } & \multirow{3}{*}{$p$-Value * } \\
\hline & & & & \multicolumn{2}{|c|}{ No $(n=92)$} & \multicolumn{2}{|c|}{ Yes $(n=194)$} & \\
\hline & & $n$ & $\%$ & $n$ & $\%$ & $N$ & $\%$ & \\
\hline $\operatorname{Sex}(n=286)$ & $\mathrm{M}$ & 194 & 67.8 & 52 & 56.5 & 142 & 73.2 & 0.005 \\
\hline \multirow{4}{*}{ BMI $(n=194)$} & $<18.5$ & - & - & - & - & - & - & \multirow{4}{*}{0.011} \\
\hline & $18.5-24.9$ & 22 & 11.3 & 11 & 19.3 & 11 & 8.03 & \\
\hline & $25.0-29.9$ & 78 & 40.2 & 15 & 26.3 & 63 & 45.9 & \\
\hline & $\geq 30$ & 94 & 48.5 & 31 & 54.4 & 63 & 45.9 & \\
\hline \multirow{2}{*}{$\operatorname{NRS}(n=286)$} & $<3$ & 28 & 9.8 & 9 & 9.8 & 19 & 9.8 & \multirow{2}{*}{0.991} \\
\hline & $\geq 3$ & 258 & 90.2 & 83 & 90.2 & 175 & 90.2 & \\
\hline $\mathrm{HF}(n=286)$ & Yes & 19 & 6.64 & 2 & 2.2 & 17 & 8.8 & 0.037 \\
\hline $\mathrm{HT}(n=286)$ & Yes & 145 & 50.7 & 38 & 41.3 & 107 & 55.2 & 0.029 \\
\hline $\mathrm{DM}(n=286)$ & Yes & 92 & 32.2 & 25 & 27.2 & 67 & 34.5 & 0.214 \\
\hline $\operatorname{CVD}(n=286)$ & Yes & 99 & 34.6 & 30 & 32.6 & 69 & 35.6 & 0.622 \\
\hline CRD $(n=286)$ & Yes & 24 & 9.4 & 6 & 6.52 & 18 & 9.3 & 0.433 \\
\hline $\mathrm{CKD}(n=286)$ & Yes & 8 & 2.8 & 1 & 1.1 & 7 & 3.6 & 0.232 \\
\hline $\mathrm{TC}(n=232)$ & $>190$ & 49 & 21.1 & 20 & 24.4 & 29 & 19.3 & 0.900 \\
\hline TGs $(n=251)$ & $>150$ & 183 & 72.9 & 58 & 73.4 & 125 & 72.7 & 0.372 \\
\hline \multicolumn{2}{|c|}{ Variables } & $\overline{\mathbf{x}}$ & SD & $\overline{\mathbf{x}}$ & SD & $\overline{\mathbf{x}}$ & SD & $p$-value $* *$ \\
\hline \multicolumn{2}{|c|}{ Age $(n=286)$} & 60.5 & 13.2 & 53.8 & 13.5 & 63.6 & 11.8 & $<0.001$ \\
\hline \multicolumn{2}{|c|}{ ICU length stay $(n=286)$} & 14.2 & 14.4 & 20.2 & 16.0 & 11.0 & 12.6 & $<0.001$ \\
\hline \multicolumn{2}{|c|}{ NRS $(n=286)$} & 3.3 & 1.1 & 3.1 & 1.1 & 3.4 & 1.1 & 0.061 \\
\hline \multicolumn{2}{|c|}{$\mathrm{BMI}(n=194)$} & 31.0 & 5.7 & 31.6 & 6.4 & 30.7 & 5.4 & 0.291 \\
\hline \multicolumn{2}{|c|}{ TGs $[\mathrm{mg} / \mathrm{dL}](n=251)$} & 250.3 & 148.3 & 236.7 & 160.5 & 256.5 & 142.5 & 0.333 \\
\hline \multicolumn{2}{|c|}{$\mathrm{TC}[\mathrm{mg} / \mathrm{dL}](n=232)$} & 144.2 & 50.7 & 155.8 & 47.9 & 137.9 & 51.2 & 0.010 \\
\hline \multicolumn{2}{|c|}{ Albumins $[\mathrm{g} / \mathrm{dL}](n=276)$} & 2.9 & 0.4 & 2.9 & 0.4 & 2.9 & 0.4 & 0.652 \\
\hline \multicolumn{2}{|c|}{ Lymphocytes [\%] $(n=271)$} & 9.3 & 10.4 & 9.4 & 7.7 & 9.3 & 11.5 & 0.981 \\
\hline \multicolumn{2}{|c|}{ Potassium $[\mathrm{mmol} / \mathrm{L}](n=280)$} & 4.4 & 0.8 & 4.3 & 0.7 & 4.5 & 0.9 & 0.092 \\
\hline \multicolumn{2}{|c|}{ Sodium $[\mathrm{mmol} / \mathrm{L}](n=280)$} & 139.6 & 5.4 & 140.2 & 4.2 & 139.2 & 5.8 & 0.141 \\
\hline \multicolumn{2}{|c|}{$\mathrm{CRP}[\mathrm{mg} / \mathrm{L}](n=281)$} & 140.1 & 100.2 & 132.7 & 87.1 & 143.5 & 105.7 & 0.400 \\
\hline \multicolumn{2}{|c|}{ PCT [ng $/ \mathrm{mL}](n=280)$} & 2.1 & 8.7 & 0.5 & 0.8 & 2.9 & 10.4 & 0.030 \\
\hline
\end{tabular}

Abbreviations: $n$, number of participants; $\bar{x}$ mean; SD, standard deviation; M, males; $p$, level of significance; BMI, body mass index; NRS, nutritional risk screening; HF, heart failure; HT, arterial hypertension; DM, diabetes mellitus; CVD, cardiovascular disease; CRD, chronic respiratory disease; CKD, chronic kidney disease; TGs, triglycerides; TC, total cholesterol; CRP, C-reactive protein; $\mathrm{PCT}$, procalcitonin; * $\chi^{2}$ test; ${ }^{* *}$-test.

\subsection{Subgroup Analysis According to BMI}

A comparison of the assessed variables between groups according to BMI is shown in Tables 2 and 3. Men showed a statistically significantly higher percentage in the BMI ranges of $18.5-24.9 \mathrm{~kg} / \mathrm{m}^{2}, 25.0-29.9$ and above $30 \mathrm{~kg} / \mathrm{m}^{2}$, compared to women. The highest percentage of deaths was observed in patients with BMI between 25.0 and $29.9 \mathrm{~kg} / \mathrm{m}^{2}$ (Table 2). The highest (statistically significant) CRP levels were observed in the group of patients with BMI between 18.5 and $24.9 \mathrm{~kg} / \mathrm{m}^{2}$. 
Table 2. Comparison of assessed parameters (qualitative variables) and BMI range (WHO criteria) values.

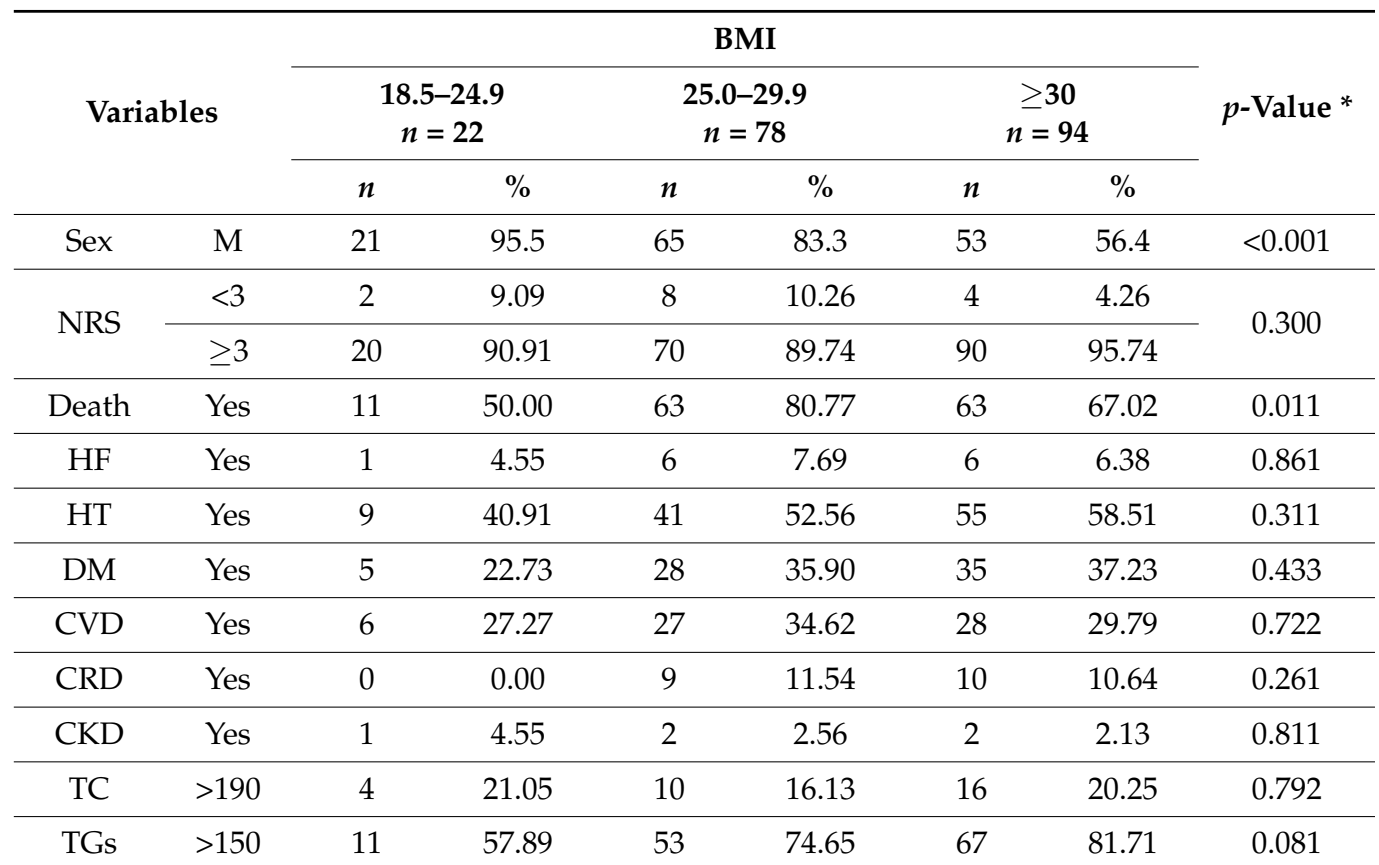

Abbreviations: $n$, number of participants; M, males; $p$, level of significance; BMI, body mass index; NRS, nutritional risk screening; HF, heart failure; HT, arterial hypertension; DM, diabetes mellitus; CVD, cardiovascular disease CRD, chronic respiratory disease; CKD, chronic kidney disease; TGs, triglycerides; TC, total cholesterol; CRP, C-reactive protein; PCT, procalcitonin; ${ }^{*} \chi^{2}$ test.

Table 3. Comparison of assessed parameters (quantitative variables) and BMI range (WHO criteria) values.

\begin{tabular}{|c|c|c|c|c|c|c|c|c|c|c|}
\hline \multirow{3}{*}{ Variables } & \multicolumn{9}{|c|}{ BMI } & \multirow{3}{*}{$p$-Value $* *$} \\
\hline & \multicolumn{3}{|c|}{$\begin{array}{c}18.5-24.9 \\
n=22\end{array}$} & \multicolumn{3}{|c|}{$\begin{array}{c}25.0-29.9 \\
n=78\end{array}$} & \multicolumn{3}{|c|}{$\begin{array}{c}\geq 30 \\
n=94\end{array}$} & \\
\hline & $n$ & $\overline{\mathbf{x}}$ & SD & $n$ & $\overline{\mathbf{x}}$ & SD & $n$ & $\overline{\mathbf{x}}$ & SD & \\
\hline Age & 22 & 56.6 & 17.2 & 78 & 62.8 & 10.0 & 94 & 60.2 & 12.2 & 0.081 \\
\hline NRS & 22 & 3.4 & 1.4 & 78 & 3.4 & 1.2 & 94 & 3.4 & 1.0 & 1.001 \\
\hline TGs [mg/dL] & 19 & 215.8 & 160.8 & 71 & 242.9 & 123.7 & 82 & 259.4 & 128.7 & 0.392 \\
\hline $\mathrm{TC}[\mathrm{mg} / \mathrm{dL}]$ & 19 & 143.1 & 46.1 & 62 & 131.2 & 50.7 & 79 & 149.7 & 48.8 & 0.091 \\
\hline Albumins [g/dL] & 21 & 3.0 & 0.4 & 76 & 2.9 & 0.5 & 91 & 3.0 & 0.4 & 0.202 \\
\hline Lymphocytes [\%] & 18 & 6.0 & 3.7 & 76 & 10.2 & 14.3 & 90 & 9.3 & 6.7 & 0.322 \\
\hline $\begin{array}{l}\text { Potassium } \\
{[\mathrm{mmol} / \mathrm{L}]}\end{array}$ & 21 & 4.4 & 0.9 & 77 & 4.5 & 0.9 & 92 & 4.3 & 0.6 & 0.281 \\
\hline Sodium [mmol/L] & 21 & 138.8 & 4.0 & 77 & 140.6 & 5.4 & 92 & 139.4 & 5.8 & 0.242 \\
\hline CRP [mg/L] & 21 & 183.5 & 115.9 & 77 & 122.3 & 100.8 & 92 & 133.8 & 89.5 & 0.040 \\
\hline PCT [ng/mL] & 21 & 2.5 & 8.6 & 76 & 1.7 & 4.7 & 92 & 1.9 & 9.3 & 0.913 \\
\hline
\end{tabular}

Abbreviations: $n$, number of participants; $\overline{\mathrm{x}}$, mean; SD, standard deviation; $p$, level of significance; BMI, body mass index; TGs, triglycerides; TC, total cholesterol; CRP, C-reactive protein; PCT, procalcitonin; ** $t$-test.

\subsection{Subgroup Analysis According to NRS}

Comparisons of the assessed parameters between groups according to NRS scores are shown in Tables 4 and 5. Based on the NRS score, two groups were distinguished: NRS $<3$ and $\geq 3$. There were no statistically significant differences between the groups. 
Table 4. Comparison of assessed parameters (qualitative variables) and NRS scores.

\begin{tabular}{|c|c|c|c|c|c|c|}
\hline & & \multicolumn{4}{|c|}{ NRS } & \multirow{3}{*}{$p$-Value * } \\
\hline \multirow{2}{*}{\multicolumn{2}{|c|}{ Variables }} & \multicolumn{2}{|c|}{$\begin{array}{c}<3 \\
n=28\end{array}$} & \multicolumn{2}{|c|}{$\begin{array}{c}\geq 3 \\
n=258\end{array}$} & \\
\hline & & $n$ & $\%$ & $n$ & $\%$ & \\
\hline Sex & M & 18 & 64.39 & 176 & 68.22 & 0.671 \\
\hline \multirow{3}{*}{ BMI } & $<18.5$ & 2 & 14.29 & 20 & 11.11 & \multirow{3}{*}{0.301} \\
\hline & $18.5-24.9$ & 8 & 57.14 & 70 & 38.89 & \\
\hline & $25.0-29.9$ & 4 & 28.57 & 90 & 50.00 & \\
\hline Death & Yes & 19 & 67.86 & 175 & 67.83 & 0.994 \\
\hline $\mathrm{HF}$ & Yes & 1 & 3.57 & 18 & 6.98 & 0.493 \\
\hline $\mathrm{HT}$ & Yes & 11 & 39.29 & 134 & 51.94 & 0.202 \\
\hline DM & Yes & 9 & 32.14 & 83 & 32.17 & 0.992 \\
\hline CVD & Yes & 13 & 46.43 & 86 & 33.33 & 0.171 \\
\hline CRD & Yes & 4 & 14.29 & 20 & 7.75 & 0.244 \\
\hline CKD & Yes & 0 & 0.00 & 8 & 3.10 & 0.343 \\
\hline TC & $>190$ & 7 & 30.43 & 42 & 20.10 & 0.253 \\
\hline TGs & $>150$ & 17 & 68.00 & 166 & 73.45 & 0.561 \\
\hline
\end{tabular}

Abbreviations: $n$, number of participants; M, males; $p$, level of significance; BMI, body mass index; NRS, nutritional risk screening; HF, heart failure; HT, arterial hypertension; DM, diabetes mellitus; CVD, cardiovascular disease; CRD, chronic respiratory disease; CKD, chronic kidney disease; TGs, triglycerides; TC, total cholesterol; CRP, C-reactive protein; $\mathrm{PCT}$, procalcitonin; ${ }^{*} \chi^{2}$ test.

Table 5. Comparison of assessed parameters (quantitative variables) and NRS scores.

\begin{tabular}{|c|c|c|c|c|c|c|c|}
\hline \multirow{3}{*}{ Variables } & \multicolumn{6}{|c|}{ NRS } & \multirow{3}{*}{$p$-Value $* *$} \\
\hline & \multicolumn{3}{|c|}{$\begin{array}{c}<3 \\
n=28\end{array}$} & \multicolumn{3}{|c|}{$\begin{array}{c}\geq 3 \\
n=258\end{array}$} & \\
\hline & $n$ & $\overline{\mathbf{x}}$ & SD & $n$ & $\overline{\mathbf{x}}$ & SD & \\
\hline Age & 28 & 57.3 & 13.2 & 258 & 60.8 & 13.2 & 0.181 \\
\hline BMI & 14 & 29.6 & 6.7 & 180 & 31.1 & 5.7 & 0.362 \\
\hline Height [cm] & 13 & 174.5 & 8.2 & 180 & 174.8 & 9.1 & 0.901 \\
\hline Body Mass [kg] & 13 & 85.6 & 13.1 & 181 & 94.8 & 17.7 & 0.071 \\
\hline TGs [mg/dL] & 25 & 240.1 & 150.0 & 226 & 251.4 & 148.5 & 0.722 \\
\hline $\mathrm{TC}[\mathrm{mg} / \mathrm{dL}]$ & 23 & 148.9 & 61.1 & 209 & 143.7 & 49.6 & 0.644 \\
\hline Albumins [g/dL] & 27 & 2.8 & 0.5 & 249 & 2.9 & 0.4 & 0.071 \\
\hline Lymphocytes [\%] & 26 & 8.2 & 3.9 & 245 & 9.5 & 10.9 & 0.551 \\
\hline Potassium [mmol/L] & 27 & 4.6 & 1.1 & 253 & 4.4 & 0.8 & 0.242 \\
\hline Sodium [mmol/L] & 27 & 141.1 & 5.4 & 253 & 139.4 & 5.4 & 0.121 \\
\hline $\mathrm{CRP}[\mathrm{mg} / \mathrm{L}]$ & 28 & 123.5 & 93.7 & 253 & 141.9 & 100.9 & 0.364 \\
\hline PCT [ng/mL] & 28 & 3.6 & 16.0 & 252 & 2.0 & 7.5 & 0.351 \\
\hline
\end{tabular}

Abbreviations: $n$, number of participants; $\overline{\mathrm{x}}$, mean; SD, standard deviation; $p$, level of significance; BMI, body mass index; TGs, triglycerides; TC, total cholesterol; CRP, C-reactive protein; PCT, procalcitonin; ** $t$-test.

\subsection{Survival Analysis}

Survival analysis is shown in Kaplan-Meier survival curves (Figure 1). The median survival was 14 days (Table 6$)$. Total survival was $32.2 \%(n=92)$. 


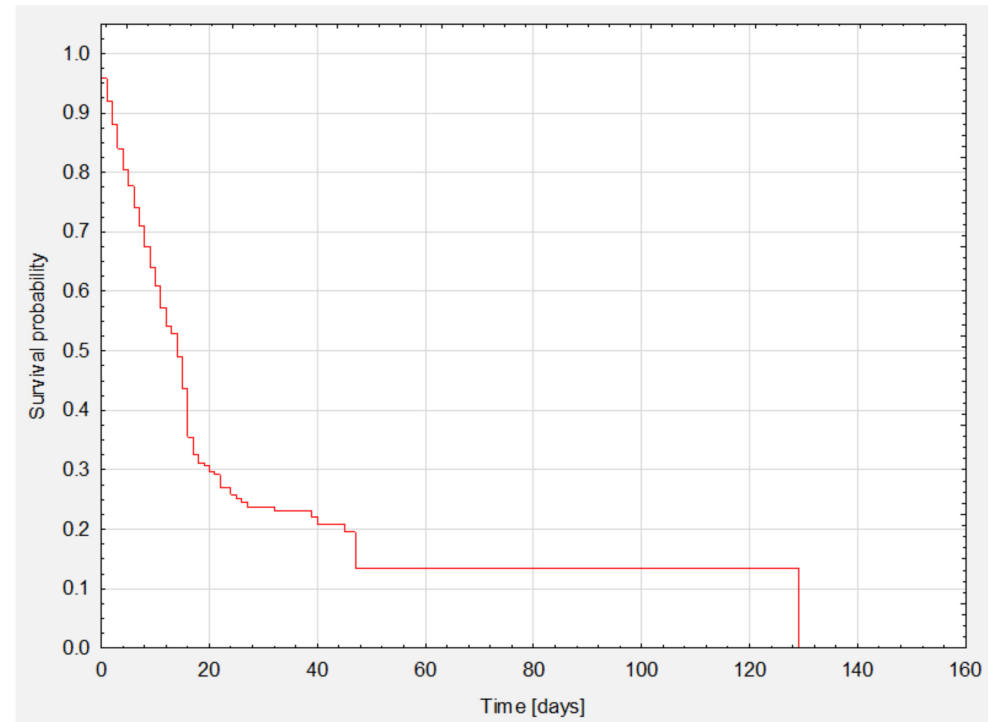

Figure 1. Analysis of survival of the whole group.

Table 6. Survival time.

\begin{tabular}{ccc}
\hline & & Survival Time [Days] \\
\hline \multirow{3}{*}{ Percentiles } & 25 percentiles (lower quartile) & 6.0 \\
\cline { 2 - 3 } & 50 percentiles (median) & 14.3 \\
\cline { 2 - 3 } & 75 percentiles (upper quartile) & 25.3 \\
\hline
\end{tabular}

\subsection{Survival Analysis_Group Comparisons}

A comparison of survival curves was performed according to BMI and NRS. There were no statistically significant differences (Table 7, Figures 2 and 3).

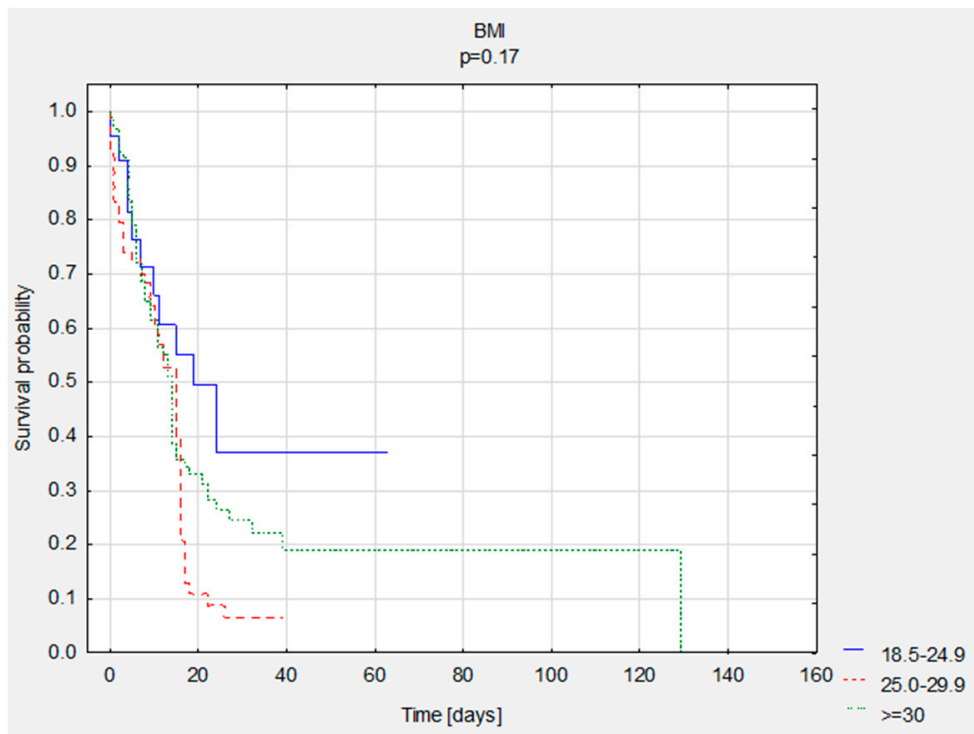

Figure 2. Comparison of survival curves according to BMI scores. 
Table 7. Descriptive statistics for survival time, number of deaths and survival according to BMI and NRS scores.

\begin{tabular}{ccccccc}
\hline & & \multicolumn{5}{c}{ Descriptive Statistics } \\
\cline { 2 - 7 } & & Me & $\overline{\mathbf{x}}$ & SD & $n$-Death & $n$-Survivors \\
\hline \multirow{3}{*}{ BMI } & $<18.5$ & - & - & - & - & - \\
\cline { 2 - 7 } & $18.5-24.9$ & 13.0 & 18.0 & 17.8 & 11 & 11 \\
\cline { 2 - 7 } & $25.0-29.9$ & 11.5 & 10.8 & 8.2 & 63 & 15 \\
\cline { 2 - 7 } & $\geq 30$ & 11.0 & 14.7 & 16.6 & 63 & 31 \\
\hline \multirow{2}{*}{ NRS } & $<3$ & 10.0 & 13.2 & 14.8 & 19 & 9 \\
& $>3$ & 11.0 & 14.4 & 14.3 & 175 & 83 \\
\hline
\end{tabular}

Abbreviations: $n$, number of participants; Me, median; $\bar{x}$, mean; SD, standard deviation; BMI, body mass index NRS, nutritional risk screening.

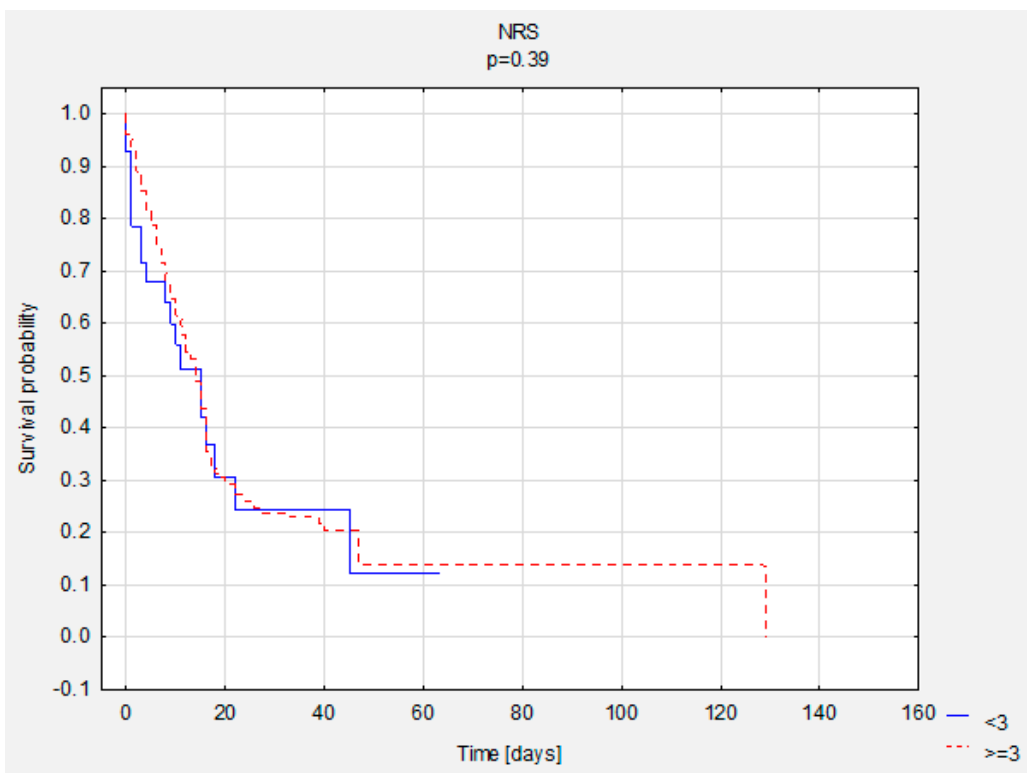

Figure 3. Comparison of survival curves according to NRS scores.

Assessment of the influence of selected variables on mortality is shown in Table 8 (Cox proportional hazards regression). It was observed that the risk of death increased in the group with BMI in the range of $25.0-29.9(\mathrm{HR}=2.18 ; p=0.010)$. Taking the quantitative variables into account, the risk of death was lower in patients with higher levels of TC $(\mathrm{HR}=0.996 ; p=0.034)$ and sodium $(\mathrm{HR}=0.97 ; p=0.033)$. However, age $(\mathrm{HR}=1.03$; $p<0.001)$, NRS $(\mathrm{HR}=1.18 ; p=0.019)$, high potassium $(\mathrm{HR}=1.34 ; p=0.002)$ and PCT $(\mathrm{HR}=1.04 ; p<0.001)$ also affected mortality.

Table 8. Assessment of the influence of variables on mortality: the Cox proportional hazards regression model, a single model.

\begin{tabular}{cccccc}
\hline & & $p$-Value & HR & $\begin{array}{c}\text { 95\% CI HR } \\
\text { (Lower) }\end{array}$ & $\begin{array}{c}\text { 95\% CI HR } \\
\text { (Upper) }\end{array}$ \\
\hline Sex $(n=286)$ & $\mathrm{M}$ & 0.451 & 1.13 & 0.82 & 1.56 \\
\hline \multirow{3}{*}{ BMI $(n=194)$} & $18.5-24.9$ & \multicolumn{3}{c}{ Ref. } \\
\cline { 2 - 6 } & $25.0-29.9$ & 0.010 & 2.18 & 1.14 & 4.16 \\
\cline { 2 - 6 } & $\geq 30$ & 0.662 & 1.62 & 0.85 & 3.07 \\
\hline \multirow{2}{*}{ NRS $(n=286)$} & $<3$ & & \multicolumn{3}{c}{ Ref. } \\
\cline { 2 - 6 } & $\geq 3$ & 0.661 & 0.90 & & \\
\hline
\end{tabular}


Table 8. Cont.

\begin{tabular}{|c|c|c|c|c|c|}
\hline & & $p$-Value & HR & $\begin{array}{l}\text { 95\% CI HR } \\
\text { (Lower) }\end{array}$ & $\begin{array}{c}95 \% \text { CI HR } \\
\text { (Upper) }\end{array}$ \\
\hline $\operatorname{HF}(n=286)$ & Yes & 0.281 & 1.32 & 0.79 & 2.21 \\
\hline $\mathrm{HT}(n=286)$ & Yes & 0.733 & 1.05 & 0.79 & 1.40 \\
\hline $\mathrm{DM}(n=286)$ & No & 0.344 & 1.15 & 0.86 & 1.55 \\
\hline $\operatorname{CVD}(n=286)$ & Yes & 0.941 & 1.01 & 0.75 & 1.36 \\
\hline CRD $(n=286)$ & Yes & 0.080 & 1.55 & 0.95 & 2.52 \\
\hline $\operatorname{CKD}(n=286)$ & Yes & 0.641 & 1.20 & 0.56 & 2.55 \\
\hline TGs $(n=251)$ & $>150$ & 0.671 & 1.08 & 0.77 & 1.51 \\
\hline $\mathrm{TC}(n=232)$ & $>190$ & 0.184 & 0.76 & 0.51 & 1.14 \\
\hline \multicolumn{6}{|c|}{ Variables } \\
\hline \multicolumn{2}{|c|}{ Age $(n=286)$} & 0.000 & 1.03 & 1.02 & 1.04 \\
\hline \multicolumn{2}{|c|}{ NRS $(n=286)$} & 0.019 & 1.18 & 1.03 & 1.35 \\
\hline \multicolumn{2}{|c|}{$\mathrm{BMI}(n=194)$} & 0.522 & 0.99 & 0.96 & 1.02 \\
\hline \multicolumn{2}{|c|}{ Height $[\mathrm{cm}](n=193)$} & 0.762 & 1.00 & 0.98 & 1.02 \\
\hline \multicolumn{2}{|c|}{ Body Mass $[\mathrm{kg}](n=194)$} & 0.733 & 1.00 & 0.99 & 1.01 \\
\hline \multicolumn{2}{|c|}{ TGs $[\mathrm{mg} / \mathrm{dL}](n=251)$} & 0.844 & 1.00 & 1.00 & 1.00 \\
\hline \multicolumn{2}{|c|}{$\mathrm{TC}[\mathrm{mg} / \mathrm{dL}](n=232)$} & 0.034 & 1.00 & 0.99 & 1.00 \\
\hline \multicolumn{2}{|c|}{ Albumins $[\mathrm{g} / \mathrm{dL}](n=276)$} & 0.844 & 1.04 & 0.74 & 1.44 \\
\hline \multicolumn{2}{|c|}{ Lymphocytes [\%] $(n=271)$} & 0.811 & 1.00 & 0.99 & 1.02 \\
\hline \multicolumn{2}{|c|}{ Potassium $[\mathrm{mmol} / \mathrm{L}](n=280)$} & 0.002 & 1.34 & 1.11 & 1.61 \\
\hline \multicolumn{2}{|c|}{ Sodium [mmol/L] $(n=280)$} & 0.033 & 0.97 & 0.95 & 1.00 \\
\hline \multicolumn{2}{|c|}{$\mathrm{CRP}[\mathrm{mg} / \mathrm{L}](n=281)$} & 0.283 & 1.00 & 1.00 & 1.00 \\
\hline \multicolumn{2}{|c|}{ PCT $[\mathrm{ng} / \mathrm{mL}](n=280)$} & 0.000 & 1.04 & 1.03 & 1.05 \\
\hline
\end{tabular}

Abbreviations: $n$, number of participants; M, males; HR, hazard ratio; CI, confidence interval; $p$, level of significance; BMI, body mass index; NRS, nutritional risk screening; HF, heart failure; HT, arterial hypertension $\mathrm{DM}$, diabetes mellitus; CVD, cardiovascular disease; CRD, chronic respiratory disease; CKD, chronic kidney disease; TGs, triglycerides; TC, total cholesterol; CRP, C-reactive protein; PCT, procalcitonin.

Variables were included in the multivariate model in accordance with the adopted criteria. The criteria included the outcome of $p<0.30$ in a univariate model, a lack of correlation of variables, and clinical recommendations. The following variables were included in the model: BMI (categories), HF, TC (quantitatively), age, NRS (quantitatively), potassium, sodium, CKD, CRP and PCT (Table 9).

The multivariate analysis showed that age $(\mathrm{HR}=1.03, p \leq 0.001)$, potassium $(\mathrm{HR}=1.40$, $p=0.023)$, PCT $(\mathrm{HR}=0.10, p<0.001)$ and BMI 25.00-29.99 correlated with mortality $(\mathrm{HR}=2.13, p=0.038)$. Table 9 shows statistically significant variables. 
Table 9. Assessment of the influence of variables on mortality: the Cox proportional hazards regression model, a multivariate model.

\begin{tabular}{|c|c|c|c|c|c|c|c|c|}
\hline \multicolumn{2}{|c|}{$n=153$} & Beta & Standard Error & Chi-Square & $p$-Value & HR & 95\% CI HR (Lower) & 95\% CI HR (Upper) \\
\hline \multicolumn{2}{|c|}{ Age } & 0.03 & 0.01 & 11.2 & 0.001 & 1.03 & 1.01 & 1.05 \\
\hline \multicolumn{2}{|c|}{ Potassium $[\mathrm{mmol} / \mathrm{L}]$} & 0.34 & 0.15 & 5.2 & 0.023 & 1.40 & 1.05 & 1.88 \\
\hline \multicolumn{2}{|c|}{ PCT [ng/mL] } & 0.09 & 0.02 & 23.5 & $<0.001$ & 1.10 & 1.06 & 1.14 \\
\hline \multirow{2}{*}{ BMI } & $25.0-29.9$ & 0.33 & 0.16 & 4.3 & 0.038 & 2.13 & 1.03 & 4.40 \\
\hline & $\geq 30$ & 0.09 & 0.16 & 0.3 & 0.561 & 1.68 & 0.81 & 3.47 \\
\hline
\end{tabular}

Abbreviations: $n$, number of participants; M, males; HR, hazard ratio; CI, confidence interval; $p$, level of significance; BMI, body mass index; $\mathrm{TC}$, total cholesterol, $\mathrm{PCT}$, procalcitonin.

\section{Discussion}

The nutritional status of COVID-19 patients is undoubtedly related to complications, and increased risk of death during in-patient treatment. The present study showed that patients with COVID-19 who died in the ICU were statistically significantly more likely to have comorbidities such as HF $(p=0.037)$ or HT $(p=0029)$. Nearly $68 \%$ of patients did not survive to discharge. Men died statistically significantly more often $(p=0.005)$. Other authors report between 20 and $62 \%$ of deaths during hospitalisation in the ICU and in the case of mechanically (either noninvasively or invasively) ventilated patients, from $50 \%$ to as much as $97 \%$ [15-17].

In the study group, the risk of death more than doubled $(H R=2.18)$ in patients who were overweight. The reasons for this may be that overweight patient are often not aware of their health condition because they do not have symptoms (e.g., metabolic disorders such as hypertension, insulin resistance, dyslipidemia or prediabetes, which frequently occur in overweight patients); therefore, they are less likely to undergo health examinations to diagnose their diseases, and comorbidities can lead to an increasingly severe course of, and consequent death from, COVID-19. It is widely known that both being overweight and obesity are risk factors for developing many comorbidities (including hypertension, CVD, DM2, obstructive sleep apnea) considered as risk factors for severe complications of COVID-19 [18-20]. In addition, being overweight and obesity alone may cause, e.g., chronic inflammation, which may lead to lowered immunity and lung function impairment. Jingzhou et.al. showed that being overweight was significantly associated with COVID-19 mortality at a global level [21].

Excessive body fat impedes respiratory gas exchange. Subcutaneous adipose tissue in the frontal part of the chest and at its sides increases its resistance during respiration, and, as a consequence, the patient might require higher positive pressure during mechanical ventilation [22]. It is also worth emphasising that abnormal body weight could be a problem during patient extubation. In such cases, especially in patients with obesity, there is a higher probability of upper airway obstruction and re-intubation [23]. According to Kompaniyets et al., in the United States from March to December 2020, among 34,899 patients hospitalised in the ICU for COVID-19, almost $28 \%$ were overweight and $50 \%$ were obese. The risk of death increased with increasing BMI [24]. In Europe (England), Gao et al. revealed in their prospective study that among 1601 patients in the ICU, 31\% were overweight, and 50\% were obese [25]. The results of the current study are similar. Among all inpatients, more than $40 \%$ were overweight, and nearly $50 \%$ were obese. Similar results were reported in France, where being overweight and obesity concerned $41.4 \%$ and $43.4 \%$ of ICU in-patients, respectively. In the cited study, the death rate was lower and amounted to $18.5 \%$. Interestingly, multifactorial analysis indicated a paradoxical relationship between the category of BMI and mortality. Patients whose BMI was $\leq 29 \mathrm{~kg} / \mathrm{m}^{2}(\mathrm{OR}=3.64)$ and those whose BMI was $>39 \mathrm{~kg} / \mathrm{m}^{2}(\mathrm{OR}=10.45)$ were more at risk of death compared to those with a BMI of $29-39 \mathrm{~kg} / \mathrm{m}^{2}$ [26]. Researchers point out that the risk of a severe COVID-19 disease course and invasive ventilation in the ICU is higher in patients being overweight or with obesity $[27,28]$. On the other hand, they also demonstrated that obesity is not a predictor of a higher risk of death in the ICU. A study by Zhaozhong et al. confirmed 
that there were more patients with obesity admitted to the ICU compared to those with a BMI $<30 \mathrm{~kg} / \mathrm{m}^{2}$ but, at the same time, obesity did not affect survival in these hospital units [29]. In another study, severe obesity classified as a BMI $>35 \mathrm{~kg} / \mathrm{m}^{2}$, and male sex, were independently related to the need for patient intubation and death [30]. Some studies, in this case, refer to the so-called "obesity paradox". Ironically, in patients with CVD, despite increased health risks associated with obesity, treatment outcomes were often better than in slimmer patients [31]. This can be observed, for example, in patients with HF. One of the possible explanations for this phenomenon is that the disease frequently develops in patients with obesity at a younger age, and intensive therapy, which might result in reduced mortality in this group, is provided earlier. In our study there was no significant difference regarding the age of obese and nonobese patients, but in some studies people with obesity were up to 10 years younger than individuals with normal body mass [32]. In these patients, their adipose tissue could serve as a nutrient when metabolism declines [33,34]. BMI itself is not a good indicator of obesity because it does not consider exact body composition, i.e., amount of muscle, fat distribution or water retention. However, due to its ease of use and availability, it is an integral part of the evaluation of patients with other diseases as well [35]. The index does not distinguish well between obesity phenotypes; thus, the same patient with a high BMI may be an individual with an athletic physique or sarcopenic obesity. In a recently published literature review, Dalamaga et al. confirmed that obesity and increased visceral fat were significant risk factors for poor outcomes related to COVID-19. Even though the presented study did not find any relation between obesity and the risk of death, its presence should still be considered a potential risk factor for severe complications and death.

In this study, univariate analysis revealed that the risk of death increased with the risk of malnutrition according to NRS2002 (HR =1.18). Osuna-Padilla et al. showed that nutritional risk in mechanically ventilated ICU patients was related to an increased risk of death $(\mathrm{OR}=2.4$, and was found in $66 \%$ of patients [36]. A similar result was reported by Zhang et al., where nutritional risk was found in more than $60 \%$ of hospitalised patients. The mortality rate was statistically significantly higher in that group. Moreover, those patients suffered from more comorbidities [37]. In both studies, the nutritional risk was analysed using the NUTRIC score. In this study, the risk of malnutrition occurred in $90 \%$ of patients and the screening assessment of nutritional status was performed using the validated NRS2002 questionnaire. Studies by other authors show that it is a good tool to assess the nutritional status of COVID-19 patients [38]. Retrospective and review studies show a high percentage of patients who are not only critically ill are also affected by malnutrition. This number is as high as $70 \%$. These studies also show that malnutrition risk is strongly associated with mortality [39-41]. Malnutrition results in decreased body weight, muscle weakness, impaired immunity, decreased protein levels and oxygen utilisation [42], which affects the course of the disease.

In the multivariate analysis, in addition to being overweight, increases in plasma potassium levels $(H R=1.40)$ and procalcitonin levels $(H R=1.10)$ were associated with higher mortality risk. Abnormal plasma potassium levels may be one of the symptoms of acid-base imbalance that occurs in patients with acute respiratory failure. It may also cause cardiac arrhythmia, bradyarrhythmia, complete heart block and circulatory arrest $[43,44]$. In a study concerning critically ill COVID-19 patients, Shengcong et al. found a significant increase in mortality rate in patients with potassium levels $\geq 5.0 \mathrm{mmol} / \mathrm{L}$ [45]. On the other hand, according to Perez, hypokalemia is associated with longer hospital and ICU stay but does not affect mortality [46]. Research shows that an increase in procalcitonin levels is one of the indicators of disease severity in COVID-19 patients, and is a risk factor of mortality $[47,48]$. The results of this study seem to be consistent with those published so far and indicate an association between the value of this inflammatory marker and the increased risk of in-hospital mortality (in multivariate analysis: $\mathrm{HR}=1.10 ; p<0.001$ ) $[49,50]$. In the study by Fenk et al., the risk of death increased up to fivefold $(\mathrm{OR}=5.65 ; p<0.001)$ in patients with high procalcitonin levels, indicating that this marker may be useful to reflect 
the degree of lung involvement during SARS-CoV-2 infection [51]. Leoni et al., who studied 242 COVID-19 patients hospitalised in the ICU, also found that predictors of increased mortality rate in the ICU include, among others, age, obesity and higher procalcitonin levels $(\mathrm{HR}=1.03, p=0.04)$. These predictors were independently associated with 28-day mortality [52].

\section{Study Limitations}

This study has some limitations. As it involved critically ill and mechanically ventilated (using invasive methods) patients, complete data concerning their medical history and medications could not always be obtained due to the serious nature of the situation.

Besides, we obtained statistical significance according to our classification, although other classifications could have been done. In our study, the data with no statistical significance must be taken with caution, and cannot be excluded.

The inference analysis should be interpreted with the variables we selected, and considering the whole study. We also do not have information about the time between the first symptoms of COVID-19 and hospital admission to the ICU. In some cases, NRS and BMI scores were not included in medical records. Regarding to the high proportion $(90 \%)$ of individuals with a NRS $\geq 3$, this can interfere with the results. Moreover, body composition analysis was not conducted in patients, and BMI scores may not be a reliable indicator for assessing overweight and obesity. Patients did not have their waist-to-hip ratio (WHR) measured, and data concerning central obesity based on waist circumference was not reported either. Finally, the long-term survival of COVID-19 patients could not be assessed due to restrictions on access to personal data because of the anonymity of medical records.

\section{Conclusions}

Being overweight in critically ill COVID-19 patients requiring invasive mechanical ventilation increases their risk of death significantly. Additional factors indicating a higher risk of death include the patient's age, high PCT and potassium levels measured at the time of admission to the ICU. Even though the presented study did not find any association between obesity and the risk of death, obesity should still be considered a potential risk factor for severe complications and death. The lack of confirmation of this association in this study should not be interpreted as providing a potential protective effect. The risk of malnutrition at the time of ICU admission also increases the risk of in-hospital death. Undoubtedly, studies concerning the nutritional status of COVID-19 patients hospitalised in the ICU need further investigation.

Author Contributions: Conceptualisation, M.C. and R.J.-V.; methodology, M.C. and R.J.-V.; software, M.C., S.Z.; validation, M.C., R.J.-V.; formal analysis, M.C.; investigation, M.C.; resources, M.C.; data curation, M.C.; writing—original draft preparation, M.C.; writing—review and editing, M.C., R.J.-V., S.Z., V.G.-C.; supervision, M.Z.; project administration, M.C.; funding acquisition, M.C. and M.Z., S.Z. All authors have read and agreed to the published version of the manuscript.

Funding: The study was funded by the Ministry of Science and Higher Education of Poland under the statutory grant of the Wroclaw Medical University (SUB.E140.21.108).

Institutional Review Board Statement: The study was conducted according to the guidelines of the Declaration of Helsinki and approved by the Independent Bioethics Committee of the Wroclaw Medical University (decision No. KB-229/2021).

Informed Consent Statement: Not applicable.

Data Availability Statement: The data are available by contacting the corresponding author.

Acknowledgments: There were no other contributors to the article than the authors. Certified English language services were provided.

Conflicts of Interest: The authors declare no conflict of interest. 


\section{References}

1. WHO Coronavirus (COVID-19) Dashboard. Available online: https:/ / covid19.who.int (accessed on 30 August 2021).

2. Singer, P.; Blaser, A.R.; Berger, M.M.; Alhazzani, W.; Calder, P.C.; Casaer, M.P.; Hiesmayr, M.; Mayer, K.; Montejo, J.C.; Pichard, C.; et al. ESPEN Guideline on Clinical Nutrition in the Intensive Care Unit. Clin. Nutr. 2019, 38, 48-79. [CrossRef] [PubMed]

3. Wang, D.; Hu, B.; Hu, C.; Zhu, F.; Liu, X.; Zhang, J.; Wang, B.; Xiang, H.; Cheng, Z.; Xiong, Y.; et al. Clinical Characteristics of 138 Hospitalized Patients With 2019 Novel Coronavirus-Infected Pneumonia in Wuhan, China. JAMA 2020, 323, 1061-1069. [CrossRef] [PubMed]

4. Beck, M.A.; Levander, O.A. Host Nutritional Status and Its Effect on a Viral Pathogen. J. Infect. Dis. 2000, 182 (Suppl. S1), S93-S96. [CrossRef] [PubMed]

5. James, P.T.; Ali, Z.; E Armitage, A.; Bonell, A.; Cerami, C.; Drakesmith, H.; Jobe, M.; Jones, K.S.; Liew, Z.; E Moore, S.; et al. The Role of Nutrition in COVID-19 Susceptibility and Severity of Disease: A Systematic Review. J. Nutr. 2021, 151, 1854-1878. [CrossRef] [PubMed]

6. Wei, C.; Liu, Y.; Li, Y.; Zhang, Y.; Zhong, M.; Meng, X. Evaluation of the Nutritional Status in Patients with COVID-19. J. Clin. Biochem. Nutr. 2020, 67, 116-121. [CrossRef]

7. Pironi, L.; Sasdelli, A.S.; Ravaioli, F.; Baracco, B.; Battaiola, C.; Bocedi, G.; Brodosi, L.; Leoni, L.; Mari, G.A.; Musio, A. Malnutrition and Nutritional Therapy in Patients with SARS-CoV-2 Disease. Clin. Nutr. 2021, 40, 1330-1337. [CrossRef]

8. Bedock, D.; Couffignal, J.; Lassen, P.B.; Soares, L.; Mathian, A.; Fadlallah, J.; Amoura, Z.; Oppert, J.-M.; Faucher, P. Evolution of Nutritional Status after Early Nutritional Management in COVID-19 Hospitalized Patients. Nutrients 2021, 13, 2276. [CrossRef]

9. Wolf, M.; Alladina, J.; Navarrete-Welton, A.; Shoults, B.; Brait, K.; Ziehr, D.; Malhotra, A.; Corey Hardin, C.; Hibbert, K.A. Obesity and Critical Illness in COVID-19: Respiratory Pathophysiology. Obesity 2021, 29, 870-878. [CrossRef]

10. Zhang, L.; Liu, Y. Potential Interventions for Novel Coronavirus in China: A Systematic Review. J. Med Virol. 2020, 92, 479-490. [CrossRef]

11. Cederholm, T.; Jensen, G.L.; Correia, M.I.T.D.; Gonzalez, M.C.; Fukushima, R.; Higashiguchi, T.; Baptista, G.; Barazzoni, R.; Blaauw, R.; Coats, A.J.; et al. GLIM Criteria for the Diagnosis of Malnutrition-A Consensus Report from the Global Clinical Nutrition Community. Clin. Nutr. 2019, 38, 207-217. [CrossRef]

12. Lewandowicz-Umyszkiewicz, M.; Wieczorowska-Tobis, K. Nowe kryteria diagnozowania niedożywienia. GERIATRIA 2019, 13, 101-105.

13. Barazzoni, R.; Bischoff, S.C.; Breda, J.; Wickramasinghe, K.; Krznaric, Z.; Nitzan, D.; Pirlich, M.; Singer, P. ESPEN Expert Statements and Practical Guidance for Nutritional Management of Individuals with SARS-CoV-2 Infection. Clin. Nutr. 2020, 39, 1631-1638, endorsed by the ESPEN Council. [CrossRef] [PubMed]

14. Kondrup, J.; Allison, S.P.; Elia, M.; Vellas, B.; Plauth, M. ESPEN Guidelines for Nutrition Screening 2002. Clin. Nutr. 2003, 22, 415-421. [CrossRef]

15. Myers, L.C.; Parodi, S.M.; Escobar, G.J.; Liu, V.C. Characteristics of Hospitalized Adults With COVID-19 in an Integrated Health Care System in California. JAMA 2020, 323, 2195-2198. [CrossRef] [PubMed]

16. Arentz, M.; Yim, E.; Klaff, L.; Lokhandwala, S.; Riedo, F.X.; Chong, M.; Lee, M. Characteristics and Outcomes of 21 Critically Ill Patients With COVID-19 in Washington State. JAMA 2020, 323, 1612-1614. [CrossRef]

17. Wang, Y.; Lu, X.; Li, Y.; Chen, H.; Chen, T.; Su, N.; Huang, F.; Zhou, J.; Zhang, B.; Yan, F.; et al. Clinical Course and Outcomes of 344 Intensive Care Patients with COVID-19. Am. J. Respir. Crit. Care Med. 2020, 201, 1430-1434. [CrossRef] [PubMed]

18. Dixon, A.E.; Peters, U. The Effect of Obesity on Lung Function. Expert Rev. Respir. Med. 2018, 12, 755-767. [CrossRef]

19. Cercato, C.; Fonseca, F.A. Cardiovascular Risk and Obesity. Diabetol. Metab. Syndr. 2019, 11, 74. [CrossRef]

20. Sanchis-Gomar, F.; Lavie, C.J.; Mehra, M.R.; Henry, B.M.; Lippi, G. Obesity and Outcomes in COVID-19: When an Epidemic and Pandemic Collide. Mayo Clin. Proc. 2020, 95, 1445-1453. [CrossRef]

21. Wang, J.; Sato, T.; Sakuraba, A. Worldwide Association of Lifestyle-Related Factors and COVID-19 Mortality. Ann. Med. 2021, 53, 1528-1533. [CrossRef]

22. Pelosi, P.; Croci, M.; Ravagnan, I.; Vicardi, P.; Gattinoni, L. Total Respiratory System, Lung, and Chest Wall Mechanics in Sedated-Paralyzed Postoperative Morbidly Obese Patients. Chest 1996, 109, 144-151. [CrossRef] [PubMed]

23. Hibbert, K.; Rice, M.; Malhotra, A. Obesity and ARDS. Chest 2012, 142, 785-790. [CrossRef] [PubMed]

24. Kompaniyets, L.; Goodman, A.B.; Belay, B.; Freedman, D.S.; Sucosky, M.S.; Lange, S.J.; Gundlapalli, A.V.; Boehmer, T.K.; Blanck, H.M. Body Mass Index and Risk for COVID-19-Related Hospitalization, Intensive Care Unit Admission, Invasive Mechanical Ventilation, and Death-United States, March-December 2020. MMWR. Morb. Mortal. Wkly. Rep. 2021, 70, 355-361. [CrossRef] [PubMed]

25. Gao, M.; Piernas, C.; Astbury, N.M.; Hippisley-Cox, J.; O’Rahilly, S.; Aveyard, P.; A Jebb, S. Associations between BodyMass Index and COVID-19 Severity in 6.9 Million People in England: A Prospective, Community-Based, Cohort Study. Lancet Diabetes Endocrinol. 2021, 9, 350-359. [CrossRef]

26. Dana, R.; Bannay, A.; Bourst, P.; Ziegler, C.; Losser, M.-R.; Gibot, S.; Levy, B.; Audibert, G.; Ziegler, O. Obesity and Mortality in Critically Ill COVID-19 Patients with Respiratory Failure. Int. J. Obes. 2021, 45, 2028-2037. [CrossRef]

27. Zhu, Z.; Hasegawa, K.; Ma, B.; Fujiogi, M.; Camargo, C.A.; Liang, L. Association of Obesity and Its Genetic Predisposition with the Risk of Severe COVID-19: Analysis of Population-Based Cohort Data. Metabolism 2020, 112, 154345. [CrossRef] 
28. Rottoli, M.; Bernante, P.; Belvedere, A.; Balsamo, F.; Garelli, S.; Giannella, M.; Cascavilla, A.; Tedeschi, S.; Ianniruberto, S.; Del Turco, E.R.; et al. How Important Is Obesity as a Risk Factor for Respiratory Failure, Intensive Care Admission and Death in Hospitalised COVID-19 Patients? Results from a Single Italian Centre. Eur. J. Endocrinol. 2020, 183, 389-397. [CrossRef]

29. Biscarini, S.; Colaneri, M.; Ludovisi, S.; Seminari, E.; Pieri, T.C.; Valsecchi, P.; Gallazzi, I.; Giusti, E.; Cammà, C.; Zuccaro, V.; et al. The Obesity Paradox: Analysis from the SMAtteo COvid-19 REgistry (SMACORE) Cohort. Nutr.Metab. Cardiovasc. Dis. 2020, 30, 1920-1925. [CrossRef]

30. Palaiodimos, L.; Kokkinidis, D.G.; Li, W.; Karamanis, D.; Ognibene, J.; Arora, S.; Southern, W.N.; Mantzoros, C.S. Severe Obesity, Increasing Age and Male Sex Are Independently Associated with Worse in-Hospital Outcomes, and Higher in-Hospital Mortality, in a Cohort of Patients with COVID-19 in the Bronx, New York. Metab. Clin. Exp. 2020, 108, 154262. [CrossRef]

31. Elagizi, A.; Carbone, S.; Lavie, C.J.; Mehra, M.R.; Ventura, H.O. Implications of Obesity across the Heart Failure Continuum. Prog. Cardiovasc. Dis. 2020, 63, 561-569. [CrossRef]

32. Niedziela, J.; Hudzik, B.; Niedziela, N.; Gąsior, M.; Gierlotka, M.; Wasilewski, J.; Myrda, K.; Lekston, A.; Poloński, L.; Rozentryt, P. The Obesity Paradox in Acute Coronary Syndrome: A Meta-Analysis. Eur. J. Epidemiol. 2014, 29, 801-812. [CrossRef] [PubMed]

33. Bucholz, E.M.; Beckman, A.L.; Krumholz, H.A.; Krumholz, H.M.; Beckman, A.L.; Krumholz, H.A.; Krumholz, H.M. Excess Weight and Life Expectancy after Acute Myocardial Infarction: The Obesity Paradox Reexamined. Am. Heart J. 2016, 172, 173-181. [CrossRef] [PubMed]

34. Karampela, I.; Chrysanthopoulou, E.; Christodoulatos, G.S.; Dalamaga, M. Is There an Obesity Paradox in Critical Illness? Epidemiologic and Metabolic Considerations. Curr. Obes. Rep. 2020, 9, 231-244. [CrossRef]

35. Yousufuddin, M.; Takahashi, P.Y.; Major, B.; Ahmmad, E.; Al-Zubi, H.; Peters, J.; Doyle, T.; Jensen, K.; Al Ward, R.Y.; Sharma, U.; et al. Association between Hyperlipidemia and Mortality after Incident Acute Myocardial Infarction or Acute Decompensated Heart Failure: A Propensity Score Matched Cohort Study and a Meta-Analysis. BMJ Open 2019, 9, e028638. [CrossRef] [PubMed]

36. Osuna-Padilla, I.A.; Rodríguez-Moguel, N.C.; Aguilar-Vargas, A.; Rodríguez-Llamazares, S. High Nutritional Risk Using NUTRIC-Score Is Associated with Worse Outcomes in COVID-19 Critically Ill Patients. Nutr. Hosp. 2021, 38, 540-544. [CrossRef]

37. Zhang, P.; He, Z.; Yu, G.; Peng, D.; Feng, Y.; Ling, J.; Wang, Y.; Li, S.; Bian, Y. The Modified NUTRIC Score Can Be Used for Nutritional Risk Assessment as Well as Prognosis Prediction in Critically Ill COVID-19 Patients. Clin. Nutr. 2021, 40, 534-541. [CrossRef]

38. Ali, A.; Kunugi, H. Approaches to Nutritional Screening in Patients with Coronavirus Disease 2019 (COVID-19). Int. J. Environ. Res. Public Health 2021, 18, 2772. [CrossRef]

39. Lechien, J.R.; Chiesa-Estomba, C.M.; De Siati, D.R.; Horoi, M.; Le Bon, S.D.; Rodrigues, A.; Dequanter, D.; Blecic, S.; El Afia, F.; Distinguin, L.; et al. Olfactory and Gustatory Dysfunctions as a Clinical Presentation of Mild-to-Moderate Forms of the Coronavirus Disease (COVID-19): A Multicenter European Study. Eur. Arch. Oto-Rhino-Laryngol. 2020, 277, 2251-2261. [CrossRef]

40. Lechien, J.R.; Chiesa-Estomba, C.M.; Place, S.; Van Laethem, Y.; Cabaraux, P.; Mat, Q.; Huet, K.; Plzak, J.; Horoi, M.; Hans, S.; et al. Clinical and Epidemiological Characteristics of 1420 European Patients with Mild-to-Moderate Coronavirus Disease 2019. J. Intern. Med. 2020, 288, 335-344. [CrossRef]

41. Li, G.; Zhou, C.-L.; Ba, Y.-M.; Wang, Y.-M.; Song, B.; Cheng, X.-B.; Dong, Q.-F.; Wang, L.-L.; You, S.-S. Nutritional Risk and Therapy for Severe and Critical COVID-19 Patients: A Multicenter Retrospective Observational Study. Clin. Nutr. 2021, 40, 2154-2161. [CrossRef]

42. Ljungqvist, O.; van Gossum, A.; Sanz, M.L.; de Man, F. The European Fight against Malnutrition. Clin. Nutr. 2010, 29, 149-150. [CrossRef]

43. Romano, T.G.; Correia, M.D.T.; Mendes, P.V.; Zampieri, F.G.; Maciel, A.T.; Park, M. Metabolic Acid-Base Adaptation Triggered by Acute Persistent Hypercapnia in Mechanically Ventilated Patients with Acute Respiratory Distress Syndrome. Rev. Bras. De Ter. Intensiv. 2016, 28, 19-26. [CrossRef] [PubMed]

44. McMahon, G.; Mendu, M.L.; Gibbons, F.K.; Christopher, K.B. Association between Hyperkalemia at Critical Care Initiation and Mortality. Intensiv. Care Med. 2012, 38, 1834-1842. [CrossRef] [PubMed]

45. Liu, S.; Zhang, L.; Weng, H.; Yang, F.; Jin, H.; Fan, F.; Zheng, X.; Yang, H.; Li, H.; Zhang, Y.; et al. Association Between Average Plasma Potassium Levels and 30-Day Mortality During Hospitalization in Patients with COVID-19 in Wuhan, China. Int. J. Med. Sci. 2021, 18, 736-743. [CrossRef] [PubMed]

46. Moreno-Pérez, O.; Leon-Ramirez, J.-M.; Fuertes-Kenneally, L.; Perdiguero, M.; Andres, M.; Garcia-Navarro, M.; Ruiz-Torregrosa, P.; Boix, V.; Gil, J.; Merino, E.; et al. Hypokalemia as a Sensitive Biomarker of Disease Severity and the Requirement for Invasive Mechanical Ventilation Requirement in COVID-19 Pneumonia: A Case Series of 306 Mediterranean Patients. Int. J. Infect. Dis. 2020, 100, 449-454. [CrossRef]

47. Hu, R.; Han, C.; Pei, S.; Yin, M.; Chen, X. Procalcitonin Levels in COVID-19 Patients. Int. J. Antimicrob. Agents 2020, 56, 106051. [CrossRef]

48. Liu, Z.-M.; Li, J.-P.; Wang, S.-P.; Chen, D.-Y.; Zeng, W.; Chen, S.-C.; Huang, Y.-H.; Huang, J.-L.; Long, W.; Li, M.; et al. Association of Procalcitonin Levels with the Progression and Prognosis of Hospitalized Patients with COVID-19. Int. J. Med. Sci. 2020, 17, 2468. [CrossRef]

49. Lippi, G.; Plebani, M. Procalcitonin in Patients with Severe Coronavirus Disease 2019 (COVID-19): A Meta-Analysis. Clin. Chim. Acta Int. J. Clin. Chem. 2020, 505, 190-191. [CrossRef] 
50. Zhou, F.; Yu, T.; Du, R.; Fan, G.; Liu, Y.; Liu, Z.; Xiang, J.; Wang, Y.; Song, B.; Gu, X.; et al. Clinical Course and Risk Factors for Mortality of Adult Inpatients with COVID-19 in Wuhan, China: A Retrospective Cohort Study. Lancet 2020, 395, 1054-1062. [CrossRef]

51. Feng, T.; James, A.; Doumlele, K.; White, S.; Twardzik, W.; Zahid, K.; Sattar, Z.; Foronjy, R.; Nakeshbandi, M.; Chow, L. Procalcitonin Levels in COVID-19 Patients Are Strongly Associated with Mortality and ICU Acceptance in an Underserved, Inner City Population. In Proceedings of the American Thoracic Society International Conference, San Francisco, CA, USA, $14-19$ May 2021.

52. Leoni, M.L.G.; Lombardelli, L.; Colombi, D.; Bignami, E.G.; Pergolotti, B.; Repetti, F.; Villani, M.; Bellini, V.; Rossi, T.; Halasz, G.; et al. Prediction of 28-Day Mortality in Critically Ill Patients with COVID-19: Development and Internal Validation of a Clinical Prediction Model. PLoS ONE 2021, 16, e0254550. [CrossRef] 